\title{
Implant-prosthetic Rehabilitation with and without Platform Switching: A Retrospective Clinical Cohort Study
}

\author{
Alejandro Gago-García ${ }^{1}$, Cayetana Barrilero-Martín², Pantelis Petrakakis ${ }^{3}$, Joaquín de Elio-Oliveros ${ }^{4}$, Mariano del \\ Canto-Pingarrón ${ }^{5}$, Miguel Ángel Alobera-Gracia ${ }^{6}$, Jesús Seco-Calvo ${ }^{7}$
}

\begin{abstract}
Aim: The study aimed to retrospectively compare peri-implant bone loss, prosthetic complications, and patient-reported outcome measures (PROMs) after implant-prosthetic treatment on abutments with platform switch or platform match.

Materials and methods: Records of patients, who received implant-prosthetic treatment on abutments with/without platform switch in a single dental clinic between November 2015 and November 2018, were retrospectively analyzed. Analysis was restricted to the following patient selection criteria: no need for any bone grafting procedures before/during implant placement, and no serious systemic disease. Implants were conventionally loaded with screwed prosthetic restorations after a healing period of 3 months. Crestal bone loss was measured by digital radiography at implant placement and after at least 2 years under functional implant loading conditions. Patient satisfaction was recorded with the visual analogue scale (VAS) at the time of the follow-up examination.

Results: Clinical records of 59 patients were available for analysis. Patients of the study cohort received in total 128 implants with different lengths and diameters according to the manufacturer's specifications. Prosthetic restorations were fixed either on abutments with platform switch (BEGO PS-UNI: $n=74 ; 57.8 \%$ ) or platform match (BEGO SUB-TEC Universal: $n=54 ; 42.2 \%$ ). No implant was lost and no failure of prosthetic restoration was recorded during follow-up, except for prosthetic screw loosening in 32 implants (25.0\%). Abutment type and location (maxilla vs mandible) had a significant impact on peri-implant bone loss $(\mathrm{OR}=3.4 ; 2.8)$. A significant reduced rate of bone loss was observed at implant sites, provided with abutments according to the platform switch concept (35.1 vs $64.8 \%)$. No significant correlation was recorded between less bone loss and a higher patient satisfaction, while loosening of the prosthetic screw was significantly associated with lower satisfaction scores. Conclusion: BEGO PS-UNI abutments with a platform switch design revealed significant less crestal bone loss after a mean observation period of 20.8 months.

Clinical significance: Abutments with a platform switch design may lead to less peri-implant bone loss. In order to maintain a higher patient satisfaction, clinicians should focus on the quality of the implant-prosthetic connection in screwed restorations.
\end{abstract}

Keywords: Crestal bone loss, Dental abutments, Implants, Insertion torque, Patient satisfaction, Platform switching, Screw loosening.

The Journal of Contemporary Dental Practice (2021): 10.5005/jp-journals-10024-3181

\section{INTRODUCTION}

Peri-implant tissue stability is of high importance for successful implant treatment. Therefore, maintaining stable bone levels around dental implants in the short and long terms is the main objective in dental implantology. Besides clinical evaluation of implant mobility, radiological assessment of crestal bone level change after implant placement is one key determinant to define implant success. ${ }^{1,2}$ Reasons for peri-implant bone loss around dental implants are still in debate and not yet fully understood. ${ }^{3}$ Jaw volume, bone quality, and implant overload were discussed as major determinants for late implant failures already 20 years ago. ${ }^{1}$ In scientific literature, a large number of studies focus on the platformswitch/platform-shift concept, which has gained popularity over the last years. ${ }^{4}$ This concept is based on the consideration that an inward shifting of the implant-abutment interface, combined with an internal implant-abutment connection, will lead to less peri-implant bone loss, related to a matching connection between dental implant and abutment. This beneficial effect on peri-implant bone preservation is supposed to be due to biomechanical and microbiological reasons. Firstly, platform shifting will lead to a shift of stress concentration away from the crestal bone interface, directing occlusal forces along the long axis of the implant., Secondly, the gap between implant and abutment junction is supposed to comprise high numbers of inflammatory cell infiltrates,
${ }^{1,2,4-6}$ Centre for Continuing Education of the College of Dental Surgeons and Stomatologists of León, Spain

${ }^{3}$ Department of Nursing and Physical Therapy, Institute of Biomedicine (IBIOMED), University of León, León, Spain

${ }^{7}$ Department of Public Health Dentistry of the Authority of the Rhein-Erft-District, Duesseldorf, North Rhine-Westphalia, Germany

Corresponding Author: Alejandro Gago-García, Centre for Continuing Education of the College of Dental Surgeons and Stomatologists of León, Spain, Phone: +34 669198249, e-mail: alejandrogagogarcia@ gmail.com

How to cite this article: Gago-García A, Barrilero-Martín C, Petrakakis P, et al. Implant-prosthetic Rehabilitation with and without Platform Switching: A Retrospective Clinical Cohort Study. J Contemp Dent Pract 2021;22(9):1041-1047.

Source of support: Nil

Conflict of interest: None

probably causing bone resorption through tissue inflammation. ${ }^{7}$ From a biological point of view and as a key feature of the platform shift-concept, inward shifting and relocation of the implantabutment junction shall keep the inflammatory microbiota away from the crestal peri-implant bone, thus preventing peri-implant disease. ${ }^{8}$ Insights of two systematic reviews exhibited significant 
less crestal bone resorption, when implants were placed according to the platform switch concept. ${ }^{9,10}$ Wear and corrosion of titanium implants, called tribocorrosion, has been discussed as another issue on peri-implant bone loss. Degradation of dental implants may have cytotoxic potential for peri-implant tissues, leading to activation of immune cell mediators and subsequent bone loss around dental implants. ${ }^{11-13}$

Patient-reported outcome measures (PROMs) are increasingly gaining attention in dentistry. Patient's expectations seem to be very high with respect to aesthetics, as well as to functional aspects. ${ }^{14,15}$ Thus, history of complication with implant-prosthetic restorations will lead to a reduced patient satisfaction and poorer reporting on oral health-related quality of life. ${ }^{16}$ The aim of this retrospective clinical cohort study was to compare two different abutment systems without/with a platform switch concept in terms of its effects on radiological visible crestal bone loss around dental implants, prosthetic complications, and PROMs.

\section{Materials and Methods}

\section{Patients}

Consecutive patients of a single dental clinic with the need for implant-prosthetic treatment were treated between November 2015 and November 2018 either with BEGO Semados S implants and SUB-TEC Universal abutments or with BEGO Semados SC implants and PS-UNI abutments (BEGO Implant Systems, Germany). Patients were retrospectively selected according to the following criteria: no need for any bone grafting procedures before/during implant placement, and no serious systemic condition worse than grade II according to the ASA-classification system. ${ }^{17}$ Fifty-nine patients fulfilled the criteria of inclusion. Thirty patients had been provided with PS-UNI abutments, while 29 patients had received SUB-TEC abutments. Before treatment, written informed consent was obtained from all patients according to the ethical guidelines of the Declaration of Helsinki (version 2013), and the study was approved by the ethical committee of the University of Léon, Spain (UE2016/679). Patient satisfaction was recorded at the follow-up examination by the visual analogue scale (VAS). VAS records were subsequently analyzed.

\section{Implant System and Abutments}

BEGO S-line implants were made of grade IV titanium and have a homogenous, highly purified surface. The implants comprised a selftapered design with a machined collar and an internal hexagonal connection for a precise fit at the implant-abutment interface. BEGO SUB-TEC Universal and PS-UNI abutments were made of a titanium base and had a wax-up sleeve. While BEGO S implants and SUB-TEC abutments comprised a platform match design, BEGO SC implants and PS-UNI abutments were designed according to the platformswitch concept at the implant-abutment connection.

\section{Radiological Diagnostics}

Three-dimensional radiological assessments (Carestream 8100 3D, Carestream Dental LLC, Atlanta, USA) were performed before implantation, in order to determine bone density and implant location.

One panoramic radiography was taken immediately after surgical treatment for the assessment of the implant's location, and a second panoramic radiography was taken at the follow-up examination after a mean period of 20.4 months (Cranex Novus, Soredex, Finland). Bone quality was determined according to the classification of Misch. ${ }^{18,19}$ Radiological follow-up examinations were performed 1 or 2 years after implant loading by panoramic radiography in dependence on the patient's temporal availability. Measurement of crestal bone loss was performed digitally with the Digora Soredex software (KaVo, Germany). Bone loss was subdivided into four classes (bone loss $=0.0 \mathrm{~mm}$, bone loss $>0.0 \leq 1.0 \mathrm{~mm},>1.0 \leq 2.0 \mathrm{~mm}$, and $>2.0 \leq 3.0 \mathrm{~mm}$ ). First measurement immediately after implant insertion served as baseline value for the second measurement after follow-up. Measurements were performed by a calibrated dentist (AGG). Implant shoulder served as reference for the linear measurements mesially and distally of the implant. Bone loss was measured from the most mesial and distal point of the implant shoulder to the deepest crestal point of the peri-implant bone. The highest value of bone loss was recorded and assigned to one of the four groups. Magnification of the implants on the panoramic radiography was corrected with the known dimensions of each inserted implant by a simple recalculation method.

\section{Surgical Intervention}

Implants were inserted under local anesthesia epicrestal in healed ridges of the maxilla and mandible according to the manufacturer's surgical protocol, using drills and instruments of the BEGO Semados ${ }^{\circledR}$ S-Line Tray ${ }^{\text {Plus }}$ (BEGO Implant Systems). All implants were inserted in the maxilla without elevation of the sinus membrane, or any other augmentation procedures in both jaws. All implants were placed after deflection of a mucoperiosteal flap. All subjects were asked to comply with a pharmacological regimen of amoxicillin (875 mg TID for 7 days) or, if allergic to penicillin, clindamycin tablets (300 mg TID for 7 days), and anti-inflammatory medication (Ibuprofen $600 \mathrm{mg}$, every 6-8 hours as needed to a maximum of $3600 \mathrm{mg} /$ day). Implants were either submitted to a submerged healing protocol or provided with healing abutments (Healing Posts S/RI and PS HP, BEGO Implant Systems).

\section{Prosthetic Treatment}

Prosthetic treatment was performed for all implants in a conventional loading protocol after a healing period of 3 months. Patients were provided with fixed, screw-retained single crowns, bridges, or full-arch restorations. Prosthetic superstructures were made of a cobalt-chrome framework (Wirobond SG, BEGO, Germany) and were veneered with a layered ceramic (GC Products, Japan).

\section{Statistical Analysis and Sample Size Calculation}

Statistical analysis was performed with the MS Excel Addln, WinSTAT, version 2012.1.0.96 (Robert K Fitch) and BiAS for Windows (EpsilonVerlag), version 11.10. Test for normal distribution was performed with the Kolmogorov-Smirnov test statistics. In the case of nonparametric distribution of values, either nonparametric testing was performed (Mann-Whitney $U$ test) or a Box-Cox transformation was utilized, in order to enable calculation with mean values and testing with parametric statistics (paired $t$-test for intragroup and unpaired $t$-test for intergroup comparisons). Spearman's rank correlation tests and Pearson correlation tests were applied to analyze correlations between two different variables. KruskalWallis $H$ tests were used for multiple group comparison. Bivariate logistic regression was applied in order to calculate odds ratios (OR). Chi-squared test and Fisher's exact test were utilized for the analysis of bivariate data. Level of significance was set at $p=0.05$. Post hoc sample size calculation was performed with the $\mathrm{G}^{*}$ Power 
tool (version 3.1.9.2). With at least 30 patients in the PS-UNI group and 29 patients in the SUB-TEC group, an effect size $d$ set at 0.8 , and an alpha error set at 0.05 , we calculated a power of 0.82 , which we stated as sufficient for our investigation.

\section{Results}

\section{Patients}

Fifty-nine patients fulfilled the selection criteria. Mean follow-up period was 20.8 months. Mean patient age was 58.9 years at the beginning of the study. Thirteen patients were female $(22.0 \%)$ and 46 patients $(78.0 \%)$ were male. There was no significant statistical difference in age between both genders $(p=0.095)$. Fourteen patients (23.7\%) had mild systemic disease (e.g., diabetes and cardiovascular disorders), 9 (15.3\%) patients were smokers, and $17(28.8 \%)$ patients indicated regular intake of medication. Only three patients (5.1\%) had a history of former periodontitis.

\section{Bone Quality}

D2 bone quality was significantly more often located in the mandible $(p<0.001)$, whereas bone with a D3/D4-quality was significantly more often located in the maxilla $(p<0.001)$. No significant correlation was observed between bone quality and the extent of crestal bone loss ( $p=0.098$ ). Mean overall ISQ value at the time of implant placement was $38.6 \mathrm{~N} \mathrm{~cm}$. ISQ values were significantly higher in bone with good quality, with significant higher values in D1 bone, and D2 bone $(p<0.001)$, and on location, with significant higher mean ISQ values in the mandible (ISQ maxilla: $33.9 \mathrm{~N} \mathrm{~cm}$; ISQ mandible: $43.3 \mathrm{~N} \mathrm{~cm}, p<0.001$ ).

\section{Implants and Prosthetic Restorations}

Patients received in total 128 implants with lengths between 7.0 and $13.0 \mathrm{~mm}$ and a diameter ranging between 3.25 and $4.5 \mathrm{~mm}$. Sixty-five implants (50.8\%) were inserted in the maxilla, while 63 implants (49.2\%) were inserted in the mandible. The majority of implants were located in the posterior area of the maxilla (75.4\%) and the mandible (93.7\%).

No implant was lost, and any of the implants displayed biological complications like edema, bleeding on probing, or suppuration during follow-up.

There was no statistical correlation between ISQ values and extent of crestal bone loss $(r=0.098, p=0.134)$. When ISQ levels were compared between both jaws, a significant higher mean ISQ was recorded in the mandible $(43.3 \mathrm{~N} \mathrm{~cm})$ in relation to the maxilla $(33.9 \mathrm{~N} \mathrm{~cm})(p=0.001)$. No significant statistical difference was observed in ISQ values between female $(37.3 \mathrm{~N} \mathrm{~cm})$ or male patients $(39.2 \mathrm{~N} \mathrm{~cm})(p=0.414)$ (Table 1).

Table 1: Number of implants/patients and mean ISQ depending on the respective location (jaw) and gender

\begin{tabular}{lcc}
\hline Location & Maxilla & Mandible \\
\hline Number of implants & 65 & 63 \\
Mean ISQ $(\mathrm{N} \mathrm{cm})$ & 33.9 & 43.3 \\
Gender & Female & Male \\
Number of patients & 13 & 46 \\
Mean ISQ $(\mathrm{N} \mathrm{cm})$ & 37.3 & 39.2 \\
\hline
\end{tabular}

\section{Crestal Bone Loss and Soft Tissue Conditions}

At the respective clinical and radiological follow-up examination, all patients displayed healthy soft tissue parameters without any signs of midfacial recession, swelling, or edema of the gingiva, bleeding on probing, or suppuration. Bone loss frequency was significantly higher around implants placed in the mandible $(n=38$; $60.3 \%$ ) compared to implants placed in the maxilla ( $n=23 ; 35.4 \%)$ $(p=0.004)$ (Fig. 1). No bone loss was recorded in 67 implants (52.3\%), while 61 implants (47.7\%) displayed crestal bone loss up to $3.0 \mathrm{~mm}$.

\section{Prosthetic Treatment}

On patient level, implant-prosthetic rehabilitation revealed single crowns ( $n=22 ; 37.3 \%$ ), fixed bridges ( $n=33 ; 55.9 \%$ ), or full-arch restorations ( $n=4 ; 6.8 \%)$. Restorations were all screw-retained. No significant correlation was found between prosthetic complications and bone loss by binary logistic regression $(p=0.412$ ) (Table 2).

As for technical complications, no chipping of the veneering ceramic was observed during follow-up. Abutment screw loosening was the single prosthetic complication which occurred during follow-up period in 32 of the 128 implants (25.0\%). There was no significant correlation between the type of prosthesis and the loosening of the abutment screw $(p=0.735)$.

\section{Abutments}

Thirty patients received 74PS-UNI abutments with a platform-switch design (57.8\%), while 29 patients were provided with 54 SUB-TEC Universal platform matching abutments (42.2\%). A significantly higher portion of implants located in the mandible was provided with SUB-TEC abutments (72.2\%), whereas implants in the maxilla were more frequently provided with PS-UNI abutments (67.6\%) $(p<0.001)$. Mean follow-up periods displayed significant statistical differences between both abutment types. While mean follow-up

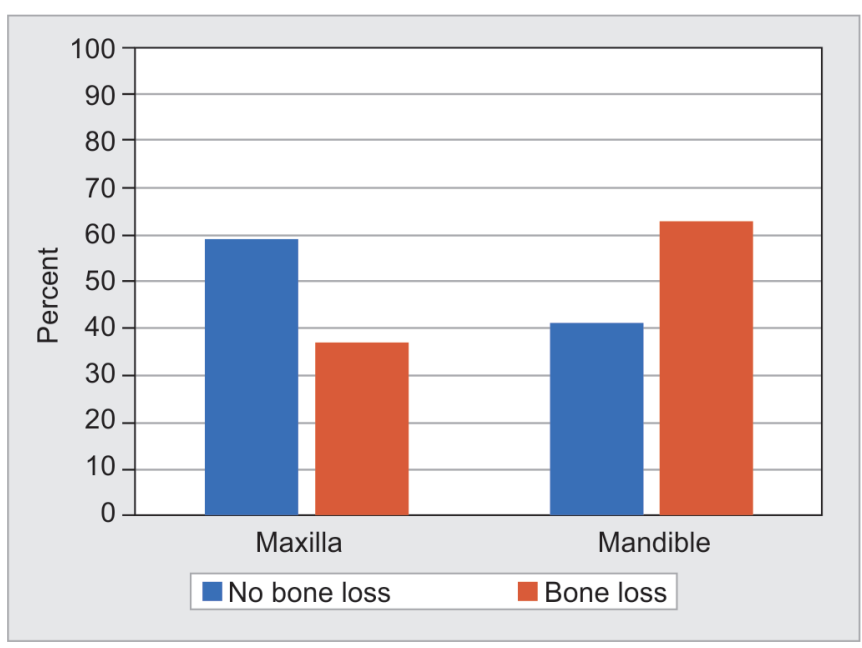

Fig. 1: Bone loss depending on implant location (jaw)

Table 2: Influence of location (jaw), abutment type, and prosthetic complications on crestal bone loss

\begin{tabular}{lccccc}
\hline Variable & Beta & SD (beta) & Wald's $p$ & OR & $95 \% \mathrm{Cl}$ \\
\hline Jaw & 1.021 & 0.368 & 0.005 & 2.8 & $1.3-5.7$ \\
Abutment type & 1.224 & 0.377 & 0.001 & 3.4 & $1.6-7.1$ \\
Prosthetic complication & 0.292 & 0.412 & 0.479 & 1.3 & $0.6-3.0$ \\
\hline
\end{tabular}


Implant-prosthetic Rehabilitation with and without Platform Switching

comprised 13.8 months for PS-UNI abutments, mean follow-up duration for SUB-TEC Universal abutments was 30.4 months $(p<0.001)$. Due to the acquisition of categorical data concerning bone-level changes, peri-implant bone loss was transferred into bivariate outcome parameters (bone loss yes/no). Significantly more implants with no signs of bone loss were recorded with PS-UNI abutments ( $p<0.001$ ) (Fig. 2; Table 3). Binary logistic regression revealed an OR of 3.4 for a higher number of implants with bone loss around SUB-TEC Universal abutments, in relation to implants with PS-UNI abutments. Location was the second influencing parameter, revealing an OR of 2.8 for radiologically visible bone loss around implants, inserted in the mandible (Table 2). There was no significant difference concerning incidence of abutment screw loosening and abutment type ( $p=0.535$, Chi-squared test). No significant difference was recorded in ISQ between both study groups (PS-UNI: $38.7 \mathrm{~N} \mathrm{~cm}$; SUB-TEC: $38.3 \mathrm{~N} \mathrm{~cm})(p=0.597)$.

\section{Patient Satisfaction}

Analysis of the VAS records displayed a high patient satisfaction, revealing VAS scores from 7 to 10 . There was no significant correlation between gender $(r=-0.042, p=0.375)$, or age $(r=0.031$, $p=0.407)$, and patient satisfaction.

Complication events obviously had a significant influence on patient satisfaction ( $p=0.001$ ). Patients with screw loosening were more prone to rate the therapy with a score equal to 9 or less. In cases with no complication events, patients were significantly more likely to rate their state of satisfaction significantly more often with a VAS score equal to 9 or 10 (Table 4).

\section{Discussion}

Crestal bone level stability has been defined as one important criterion to define implant success. ${ }^{2}$ Extent of peri-implant bone

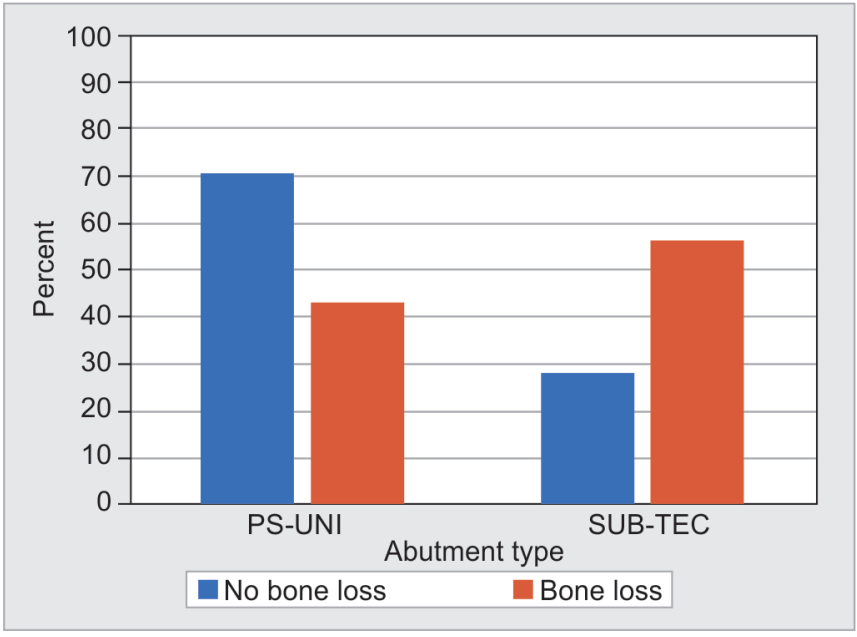

Fig. 2: Bone loss depending on abutment type

Table 3: Abutment type and bone loss

\begin{tabular}{|c|c|c|c|c|c|c|c|c|}
\hline \multirow[b]{3}{*}{ Abutment } & \multicolumn{4}{|c|}{ PS-UNI $(n=74)$} & \multicolumn{4}{|c|}{ SUB-TEC $(n=54)$} \\
\hline & \multicolumn{2}{|c|}{ Yes } & \multicolumn{2}{|c|}{ No } & \multicolumn{2}{|c|}{ Yes } & \multicolumn{2}{|c|}{ No } \\
\hline & $N$ & $\%$ & $N$ & $\%$ & $N$ & $\%$ & $N$ & $\%$ \\
\hline Bone loss & 26 & 35.1 & 48 & 64.9 & 35 & 64.8 & 19 & 35.2 \\
\hline
\end{tabular}

Table 4: Patient satisfaction scores depending on screw loosening as prosthetic complication

\begin{tabular}{lccccccccc}
\hline \multicolumn{8}{c}{ Patient satisfaction and prosthetic complications } \\
\cline { 2 - 10 } Score & \multicolumn{1}{c}{7} & \multicolumn{1}{c}{8} & & 9 & \multicolumn{2}{c}{10} \\
\hline No complications & 1 & 1.0 & 19 & 19.8 & 39 & 41.3 & 37 & 38.5 \\
& $N$ & $\%$ & $N$ & $\%$ & $N$ & $\%$ & $N$ & $\%$ \\
Complications & 3 & 9.4 & 11 & 34.4 & 16 & 50.0 & 2 & 6.3 \\
\hline
\end{tabular}

loss is highly dependent on the stability and the connection design between abutment and implant. ${ }^{20}$ Implant-specific features like neck surface characteristics and connection type have high impact on biological, technical, and aesthetical outcomes in the peri-implant area. ${ }^{21}$ An internal connection design reveals significant less bone loss due to its stability and load distribution compared to external connections, as shown by a number of systematic reviews, ${ }^{22-24}$ whereas a Morse taper design obviously has the best sealing ability and a beneficial effect on biological parameters. ${ }^{23}$ Due to its high mechanical stability, a Morse taper connection is indicated for fixed partial prostheses and overdentures. ${ }^{23}$ Implant-abutment connection type obviously has no significant impact on implant's survival or complication rates, ${ }^{22}$ presenting high survival rates for both, internal and external, connections. ${ }^{24,25}$ In contrast, another investigation displayed higher survival and success rates, as well as less crestal bone loss with Morse taper connections, while internal hexagonal connections led to better aesthetical results. ${ }^{26} \mathrm{~A}$ laser-microtextured surface at the implant's neck seems to reduce the amount of marginal bone loss. ${ }^{27}$ Notwithstanding that a microtextured neck surface leads to less marginal bone loss and reduced probing depths as shown by Chen et al., ${ }^{27}$ implant-abutment connection is supposed to be of higher impact on crestal bone levels than the implant's collar surface. ${ }^{21}$ All implants utilized in our study had the same microtextured neck surface and a hexagonal internal connection. Hence, these two implant-related parameters could be excluded as influencing variables on crestal bone loss in our investigation, whereas platform switch had a significant impact on crestal bone levels, leading to significant less peri-implant bone resorption during follow-up period in relation to abutments with a platform match design. A radiologically visible vertical bone loss of less than $0.2 \mathrm{~mm} 1$ year under functional conditions after implant placement was proposed as one important criterion for implant success. ${ }^{2}$ Later published papers proposed less than $2.0 \mathrm{~mm}$ radiographically visible bone loss after 1 year of loading as one important success criterion. ${ }^{28}$ Nowadays, a higher extent of radiologically detectable bone loss around implants during the first few years in service may not be understood as peri-implant disease. ${ }^{29}$ In fact, peri-implant bone loss is supposed to be an adaptive bone response and a physiological consequence to surgical trauma during implant preparation. ${ }^{30}$ Additionally, peri-implant bone loss was supposed to be rather related to the prosthetic phase than to the healing and remodeling period of peri-implant bone after implant insertion. ${ }^{29}$ Based on these insights, a longer follow-up period may be affiliated with a higher peri-implant bone loss. In contrast, other investigations reported a major bone loss during the interval between abutment connection and placement of the prosthetic superstructure. ${ }^{31,32}$ Our investigation revealed a significant influence of implant location (maxilla vs mandible) and type of abutment on peri-implant bone 
resorption. An OR of 3.4 was identified for bone loss in the mandible, whereas an OR of 2.8 was recorded for peri-implant bone loss around implants with SUB-TEC abutments. Latter observation may serve as indicator for the beneficial effect of the platform-switch concept on marginal bone-level preservation around dental implants.

Utilization of panoramic radiography in order to measure marginal bone loss may have served as a potential source of bias due to overlay or distortion effects of the radiological recording technique. Periapical radiography is the most commonly used method for the assessment of peri-implant bone loss ${ }^{12}$ and has been described by Albrektsson et al. as an ideal technique for crestal bone loss measurement. ${ }^{33}$ Cone-beam computed tomography (CBCT) may offer additional advantage in the assessment of periimplant bone by three-dimensional analysis. ${ }^{12}$ Although periapical radiography and CBCT seem to be more suitable for crestal bone loss assessment around implants, both techniques have their limitations. ${ }^{34}$ Periapical radiographs have to be performed in a paralleling technique. ${ }^{12}$ For this reason, they require a film holder, which enables the placement of the radiological film parallel to and the radiographic tube perpendicular to the long axis of the implant. ${ }^{12}$ Standardization of this technique is limited notably in the maxilla, where radiographs have to be taken in a bisecting technique, which will lead to a potential geometric distortion and anatomical superimposition on the radiography. ${ }^{35}$ Image quality of CBCT mainly relies on the two parameters voxel size and field of view. ${ }^{12}$ Small voxels and small fields of view will lead even though to the improvement of imaging diagnostics, but seeking the most precise position of the cone by combination of both parameters is obviously associated with high radiation exposure. ${ }^{12}$ Another limitation of CBCT is the increase of metal artifacts, evoked by low energy settings, which will result in measurement uncertainty. ${ }^{34}$ We applied standardized panoramic radiography in order to avoid multiple exposures of our patients to radiation, concomitantly serving as a diagnostic tool for implant success during follow-up, and as a basis for our measurement procedure, as described in the methodological section of our investigation.

Screw-retained crowns seemed to have higher odds for prosthetic failures, like crown loosening or chipping of the veneering ceramic. ${ }^{36}$ Other retrospective studies revealed no significant impact of retention type on technical complications. ${ }^{37-39}$ Likewise, comparable results were observed in a randomized clinical trial between screwed and cemented restorations in terms of technical and biological complications. ${ }^{40}$ In our investigation, all prosthetic superstructures were screw-retained. Screw loosening was the single technical problem, occurring in $25.0 \%$ of all restorations during the follow-up period. Other retrospective investigations reported similar prevalence rates of screw loosening up to $29.0 \%,{ }^{41}$ higher rates up to $53.0 \%,{ }^{42}$ or substantial lower screw loosening rates of $2.57 \% .{ }^{43}$ In contrast, results of a systematic review exhibited a higher dependency of prosthesis and retention type on the incidence of technical complications, reporting significantly higher technical complication rates with screw-retained fixed partial implant-supported prostheses. ${ }^{44}$ Higher technical complication rates were reported in the same review for fixed full-arch and partial prostheses, in relation to single-crown restorations. ${ }^{44}$ In contrast, there was no significant impact of prosthesis type on prosthesis functionality, screw loosening, respectively, in our investigation. Our observation was confirmed by the results of a systematic review, showing no significant correlation between different implantprosthetic reconstruction types and prosthetic failure rates. ${ }^{45}$
Patient satisfaction concerning results of implant-prosthetic treatment was high in our investigation, displaying only scores from 7 to 10 on the VAS. Due to the posterior location of the implant-supported dentures, functionality was the essential issue in judging patient-reported treatment success, with a lower focus on aesthetics. Authors of a whole string of investigations indicated a missing standardization in clinical research, concerning patient-related outcome measures after implant-prosthetic treatment. ${ }^{15,46-49}$ We chose the VAS to measure patient satisfaction, because this scoring system obviously provides good qualitative patient-related assessments. ${ }^{49}$ Age and gender had no significant influence on satisfaction scores in our investigation, whereas other clinical studies were either in accordance with our results concerning gender, but not with age, displaying lower satisfaction ratings in younger patients when measured with VAS. ${ }^{50}$ Another study was in accordance with our nonsignificant influence of age, but with a significant impact of gender on patient satisfaction, revealing lower satisfaction scores in women, when measured with the oral health-related quality of life (OHRQoL) scale. ${ }^{16}$ Results of a systematic review indicated higher expectations in treatment results after implant therapy in women. ${ }^{15}$ Notwithstanding the inconsistencies in gender- and age-related impact on patient satisfaction, patients who experienced complications with implant-supported dentures reported lower satisfaction scores. ${ }^{16}$ Our results were in accordance with the results of latter study, revealing significantly lower VAS in patients with a history of screw loosening.

Due to the time-dependent factor on peri-implant bone loss, a longer mean follow-up period of 30.4 months concerning SUB-TEC abutments in relation to the significant shorter mean observation period of 13.8 months with PS-UNI abutments might have acted as a confounding parameter on bone level change in our investigation. On the contrary, time dependency of periimplant bone loss remains inconclusive, due to the heterogeneity of the observations in other clinical studies. ${ }^{51}$ Another confounding factor might have been due to the unequal distribution of PS-UNI and SUB-TEC abutments, revealing a higher proportion of SUB-TEC abutments in implants placed in the mandible. Since the location of the implants in the mandible was containing higher odds for peri-implant bone loss, protective effects on peri-implant bone might not only be due to the platform-switch design, but be also influenced by the implant location as well.

\section{Conclusion}

In order to prevent peri-implant bone loss, abutments with a platform-switch design are recommended from a clinical perspective. In order to maintain a higher patient satisfaction, clinicians should also focus on the quality of the implant-prosthetic connection in screwed restorations. The findings in our investigation should be interpreted with caution due to the retrospective study design. For this reason, results of our study should be ascertained with further investigations.

\section{References}

1. Esposito M, Hirsch JM, Lekholm U, et al. Biological factors contributing to failures of osseointegrated oral implants. (I). Success criteria and epidemiology. Eur J Oral Sci 1998;106(1):527-551. DOI: 10.1046/j.0909-8836.t01-2-.x.

2. Albrektsson T, Zarb G, Worthington $P$, et al. The long-term efficacy of currently used dental implants: a review and proposed criteria 
of success. Int J Oral Maxillofac Implants 1986;1(1):11-25. PMID: 3527955.

3. Qian J, Wennerberg A, Albrektsson T. Reasons for marginal bone loss around oral implants. Clin Implant Dent Relat Res 2012;14(6):792-807. DOI: 10.1111/cid.12014.

4. Atieh MA, Ibrahim HM, Atieh AH. Platform switching for marginal bone preservation around dental implants: a systematic review and meta-analysis. J Periodontol 2010;81(10):1350-1366. DOI: 10.1902/ jop.2010.100232.

5. Sahabi M, Adibrad M, Mirhashemi FS, et al. Biomechanical effects of platform switching in two different implant systems: a threedimensional finite element analysis. J Dent (Tehran) 2013;10(4): 338-350. PMID: 24396353. PMCID: PMC3875508.

6. Maeda Y, Miura J, Taki I, et al. Biomechanical analysis on platform switching: is there any biomechanical rationale? Clin Oral Implants Res 2007;18(5):581-584. DOI: 10.1111/j.1600-0501.2007.01398.x.

7. Ericsson I, Persson LG, Berglundh T, et al. Different types of inflammatory reactions in peri-implant soft tissues. J Clin Periodontol 1995;22(3):255-261. DOI: 10.1111/j.1600-051x.1995.tb00143.x.

8. Lazzara R, Porter S. Platform switching: a new concept in implant dentistry for controlling postrestorative crestal bone level. Int J Periodontics Restorative Dent 2006;26(1):9-17. PMID: 16515092.

9. Aslam A, Ahmed B. Platform-switching to preserve peri-implant bone: a meta-analysis. J Coll Physicians Surg Pak 2016;26(4):315-319. PMID: 27097705.

10. de Medeiros RA, Pellizzer EP, Vechiato Filho AJ, et al. Evaluation of marginal bone loss of dental implants with internal or external connections and its association with other variables: a systematic review. J Prosthet Dent 2016;116(4):501-506.e5. DOI: 10.1016/ j.prosdent.2016.03.027.

11. Alrabeah GO, Knowles JC, Petridis H. Reduction of tribocorrosion products when using the platform-switching concept. J Dent Res 2018;97(9):995-1002. DOI: 10.1177/0022034518765751.

12. Naveau A, Shinmyouzu K, Moore C, et al. Etiology and measurement of peri-implant crestal bone loss (CBL). J Clin Med 2019;8(2). DOI: $10.3390 / \mathrm{jcm} 8020166$.

13. Noronha Oliveira M, Schunemann WVH, Mathew MT, et al. Can degradation products released from dental implants affect periimplant tissues? J Periodontal Res 2018;53(1):1-11. DOI: 10.1111/ jre.12479.

14. Abrahamsson KH, Wennstrom JL, Berglundh T, et al. Altered expectations on dental implant therapy; views of patients referred for treatment of peri-implantitis. Clin Oral Implants Res 2017;28(4): 437-442. DOI: 10.1111/clr.12817.

15. Korfage A, Raghoebar GM, Meijer HJA, et al. Patients' expectations of oral implants: a systematic review. Eur J Oral Implantol 2018;11(Suppl. 1): S65-S76. PMID: 30109300.

16. Canallatos JE, Hobbs GR, Bryington MS, et al. The effect of implant prosthesis complications on patient satisfaction. J Prosthet Dent 2020;123(2):269-276. DOI: 10.1016/j.prosdent.2018.11.015.

17. Saklad M. Grading of patients for surgical procedures. Anesthesiology 1941;2(3):281-284. DOI: 10.1097/00000542-194105000-00004.

18. Misch CE. Divisions of available bone in implant dentistry. Int J Oral Implantol 1990;7(1):9-17. PMID: 2103123.

19. Misch CE. Density of bone: effect on treatment plans, surgical approach, healing, and progressive bone loading. Int J Oral Implantol 1990;6(2):23-31. PMID: 2073394.

20. King GN, Hermann JS, Schoolfield JD, et al. Influence of the size of the microgap on crestal bone levels in non-submerged dental implants: a radiographic study in the canine mandible. J Periodontol 2002;73(10):1111-1117. DOI: 10.1902/jop.2002.73.10.1111.

21. Penarrocha-Diago MA, Flichy-Fernandez AJ, Alonso-Gonzalez R, et al. Influence of implant neck design and implant-abutment connection type on peri-implant health. Radiological study. Clin Oral Implants Res 2013;24(11):1192-1200. DOI: 10.1111/j.1600-0501.2012.02562.x.

22. Lemos CAA, Verri FR, Bonfante EA, et al. Comparison of external and internal implant-abutment connections for implant supported prostheses. A systematic review and meta-analysis. J Dent 2018;70: 14-22. DOI: 10.1016/j.jdent.2017.12.001.

23. Goiato MC, Pellizzer EP, da Silva EV, et al. Is the internal connection more efficient than external connection in mechanical, biological, and esthetical point of views? A systematic review. Oral Maxillofac Surg 2015;19(3):229-242. DOI: 10.1007/s10006-015-0494-5.

24. Palacios-Garzon N, Mauri-Obradors E, Rosello LX, et al. Comparison of marginal bone loss between implants with internal and external connections: a systematic review. Int J Oral Maxillofac Implants 2018;33(3):580-589. DOI: 10.11607/jomi.6190.

25. Pjetursson BE, Zarauz C, Strasding M, et al. A systematic review of the influence of the implant-abutment connection on the clinical outcomes of ceramic and metal implant abutments supporting fixed implant reconstructions. Clin Oral Implants Res 2018;29(Suppl. 18): 160-183. DOI: 10.1111/clr.13362.

26. Vetromilla BM, Brondani LP, Pereira-Cenci T, et al. Influence of different implant-abutment connection designs on the mechanical and biological behavior of single-tooth implants in the maxillary esthetic zone: a systematic review. J Prosthet Dent 2019;121(3):398-403.e3. DOI: 10.1016/j.prosdent.2018.05.007.

27. Chen Z, Zhang Y, Li J, et al. Influence of laser-microtextured surface collar on marginal bone loss and peri-implant soft tissue response: a systematic review and meta-analysis. J Periodontol 2017;88(7): 651-662. DOI: 10.1902/jop.2017.160805.

28. Misch CE, Perel ML, Wang HL, et al. Implant success, survival, and failure: the International Congress of Oral Implantologists (ICOI) Pisa Consensus Conference. Implant Dent 2008;17(1):5-15. DOI: 10.1097/ ID.0b013e3181676059.

29. Galindo-Moreno P, León-Cano A, Ortega-Oller I, et al. Marginal bone loss as success criterion in implant dentistry: beyond $2 \mathrm{~mm}$. Clin Oral Implants Res 2015;26(4):e28-e34. DOI: 10.1111/clr.12324.

30. Albrektsson T, Chrcanovic B, Östman PO, et al. Initial and long-term crestal bone responses to modern dental implants. Periodontol 2000 2017;73(1):41-50. DOI: 10.1111/prd.12176.

31. Cardaropoli G, Lekholm U, Wennström JL. Tissue alterations at implant-supported single-tooth replacements: a 1-year prospective clinical study. Clin Oral Implants Res 2006;17(2):165-171. DOI: 10.1111/j.1600-0501.2005.01210.x.

32. Tarnow DP, Cho SC, Wallace SS. The effect of inter-implant distance on the height of inter-implant bone crest. J Periodontol 2000;71(4): 546-549. DOI: 10.1902/jop.2000.71.4.546.

33. Albrektsson T, Buser D, Sennerby L. On crestal/marginal bone loss around dental implants. Int J Periodontics Restorative Dent 2013;33(1):9-11. PMID: 23342341.

34. Ritter L, Elger MC, Rothamel D, et al. Accuracy of peri-implant bone evaluation using cone beam $\mathrm{CT}$, digital intra-oral radiographs and histology. Dentomaxillofac Radiol 2014;43(6):20130088. DOI: 10.1259/ dmfr.20130088.

35. Hermann JS, Schoolfield JD, Nummikoski PV, et al. Crestal bone changes around titanium implants: a methodologic study comparing linear radiographic with histometric measurements. Int J Oral Maxillofac Implants 2001;16(4):475-485. PMID: 11515994.

36. Chrcanovic BR, Kisch J, Larsson C. Retrospective clinical evaluation of implant-supported single crowns: mean follow-up of 15 years. Clin Oral Implants Res 2019;30(7):691-701. DOI: 10.1111/clr.13454.

37. Cacaci C, Cantner F, Mucke T, et al. Clinical performance of screwretained and cemented implant-supported zirconia single crowns: 36-month results. Clin Oral Investig 2017;21(6):1953-1959. DOI: 10.1007/s00784-016-1982-1.

38. Heierle L, Wolleb K, Hammerle $\mathrm{CH}$, et al. Randomized controlled clinical trial comparing cemented versus screw-retained single crowns on customized zirconia abutments: 3-year results. Int J Prosthodont 2019;32(2):174-176. DOI: 10.11607/ijp.6080.

39. Kraus RD, Epprecht A, Hammerle CHF, et al. Cemented vs screwretained zirconia-based single implant reconstructions: a 3-year prospective randomized controlled clinical trial. Clin Implant Dent Relat Res 2019;21(4):578-585. DOI: 10.1111/cid.12735. 
40. Weigl P, Saarepera K, Hinrikus K, et al. Screw-retained monolithic zirconia vs. cemented porcelain-fused-to-metal implant crowns: a prospective randomized clinical trial in split-mouth design. Clin Oral Investig 2019;23(3):1067-1075. DOI: 10.1007/s00784-018-2531-x.

41. Korsch M, Walther W. Retrospective analysis of loosening of cementretained vs screw-retained fixed implant-supported reconstructions. Quintessence Int 2015;46(7):583-589. DOI: 10.3290/j.qi.a34077.

42. Wang JH, Judge R, Bailey D. A 5-year retrospective assay of implant treatments and complications in private practice: the restorative complications of single and short-span implant-supported fixed prostheses. Int J Prosthodont 2016;29(5):435-444. DOI: 10.11607/ ijp.4794.

43. Wittneben JG, Buser D, Salvi GE, et al. Complication and failure rates with implant-supported fixed dental prostheses and single crowns: a 10-year retrospective study. Clin Implant Dent Relat Res 2014;16(3):356-364. DOI: 10.1111/cid.12066.

44. Millen C, Bragger U, Wittneben JG. Influence of prosthesis type and retention mechanism on complications with fixed implant-supported prostheses: a systematic review applying multivariate analyses. Int J Oral Maxillofac Implants 2015;30(1):110-124. DOI: 10.11607/jomi.3607.

45. Wittneben JG, Millen C, Brägger U. Clinical performance of screwversus cement-retained fixed implant-supported reconstructions-a systematic review. Int J Oral Maxillofac Implants 2014;29(Suppl.): 84-98. DOI: 10.11607/jomi.2014suppl.g2.1.
46. Wijbenga JG, Schepers RH, Werker PM, et al. A systematic review of functional outcome and quality of life following reconstruction of maxillofacial defects using vascularized free fibula flaps and dental rehabilitation reveals poor data quality. J Plast Reconstr Aesthet Surg 2016;69(8):1024-1036. DOI: 10.1016/j.bjps.2016.05.003.

47. Wittneben JG, Wismeijer D, Bragger U, et al. Patient-reported outcome measures focusing on aesthetics of implant- and toothsupported fixed dental prostheses: a systematic review and metaanalysis. Clin Oral Implants Res 2018;29(Suppl 16):224-240. DOI: 10.1111/clr.13295.

48. Yao CJ, Cao C, Bornstein MM, et al. Patient-reported outcome measures of edentulous patients restored with implant-supported removable and fixed prostheses: a systematic review. Clin Oral Implants Res 2018;29(Suppl. 16):241-254. DOI: 10.1111/clr.13286.

49. Yao J, Tang H, Gao XL, et al. Patients' expectations to dental implant: a systematic review of the literature. Health Qual Life Outcomes 2014;12:153. DOI: 10.1186/s12955-014-0153-9.

50. Walton TR, Layton DM. Satisfaction and patient-related outcomes in 128 patients with single implant crowns in situ for up to 14 years. Int J Oral Maxillofac Implants 2017;32(3):667-674. DOI: 10.11607/jomi.5443.

51. Geraets W, Zhang L, Liu Y, et al. Annual bone loss and success rates of dental implants based on radiographic measurements. Dentomaxillofac Radiol 2014;43(7):20140007. DOI: 10.1259/ dmfr.20140007. 